\title{
Avaliação multi-informe do traço de neuroticismo em escolares
}

\author{
Multi-source assessment of neuroticism \\ trait in school children
}

\author{
Marcela MANSUR-ALVES \\ Carmen FLORES-MENDOZA \\ Francisco José $\mathbf{A B A D}^{2}$
}

\begin{abstract}
Resumo
A presente investigação avalia o traço de neuroticismo em escolares por meio de metodologia multi-informe que inclui autorrelato, heterorrelato, entrevista semiestruturada e observação comportamental. Especificamente, objetiva identificar o grau de concordância na avaliação de neuroticismo entre as várias fontes de informação utilizadas. A amostra [N=368] constitui parte do "Estudo Longitudinal de Avaliação das Competências Psicológicas das Crianças do Centro Pedagógico/Universidade Federal de Minas Gerais". Na seleção de crianças com alto e baixo neuroticismo, utilizaram-se os resultados em duas escalas de autorrelato - Big Five Questionnaire for Children eo Eysenck Personality Questionnaire - Junior. Logo, uma subamostra de 68 crianças foi submetida à avaliação multi-informe. Os resultados mostraram correlação moderada entre as escalas neuroticismo dos instrumentos de autorrelato. Não houve associação significativa entre o relato de pais e o de professores quando avaliaram neuroticismo, mas sim associações positivas entre o autorrelato e as avaliações multi-informes. As observações comportamentais não se mostraram úteis na mensuração dos traços e as razões para tais resultados são discutidas. Conclui-se que o presente estudo trouxe importantes contribuições para a literatura da área no que se refere à avaliação multi-informe do neuroticismo em crianças brasileiras.
\end{abstract}

Unitermos: Avaliação. Escolares do ensino fundamental. Neuroticismo.

\begin{abstract}
The present research evaluates the dimension of Neuroticism in school children using a multi-source design that includes self-report, parents' and teachers' reports, semi-structured interview and behavior observation. Specifically, the study intended to verify the agreement level in evaluation of Neuroticism from different sources of information. The sample [ $N=368]$ "Estudo Longitudinal de Avaliação das Competências Psicológicas das Crianças do Centro Pedagógico/Universidade Federal de Minas Gerais". Two self-report scales were used to select children with hight and low Neuroticism - Big Five Questionnaire for Children and Eysenck Personality Questionnaire - Junior. A sub sample of 68 children was submitted to a multi-source assessment. The results demonstrated moderate correlations between both Neuroticism scales used. There was not any significant association among parents' and teachers' reports when they evaluated Neuroticism, butwere found positive associations between self-report and multi-source assessment. Behavioral observations were not useful in the measurement of traits. The reasons of these results are discussed. To sum-up, this research brought up important contributions to the personality literature, regarding the Neuroticism multi-source assessment in Brazilian children.
\end{abstract}

Uniterms: Evaluation. Elementary students school. Neuroticism.

\footnotetext{
$\boldsymbol{\nabla V} \mathbf{V \nabla}$

1 Universidade Federal de Minas Gerais, Departamento de Psicologia, Laboratório de Avaliação das Diferenças Individuais. Av. Antonio Carlos, 6627, sala 4006, Campus Pampulha, 31270-901, Belo Horizonte, MG, Brasil. Correspondência para/Correspondence to: M. MANSUR-ALVES. E-mail: $<$ marcelamansuralves@yahoo.com.br>.

2 Universidad Autónoma de Madrid, Facultad de Psicologia. Madrid, España.
} 
Os pesquisadores do desenvolvimento têm explorado a possibilidade de que a estrutura da personalidade na infância possa compartilhar significativas similaridades com a estrutura dos traços na idade adulta (Caspi, Roberts \& Shiner, 2005). Em alguns estudos, os cinco fatores de personalidade (neuroticismo, extroversão, cordialidade, responsabilidade e abertura a experiências) têm sido obtidos da pré-escola à adolescência, em análises fatoriais de instrumentos de autorrelato, lista de adjetivos e de relatos de pais e professores (Barbaranelli, Caprara \& Rabasca, 1998; Halverson et al., 2003; Measelle, John, Ablow, Cowan \& Cowan, 2005; Mervielde, Buyst \& De Fruyt, 1995; Muris, Meesters \& Diederen, 2005; Shiner, 1998). De todos os fatores, o neuroticismo é o mais ubíquo (Caspi et al., 2005; Matthews, Deary \& Whiteman, 2003).

O traço de neuroticismo se caracteriza pela susceptibilidade do indivíduo às emoções negativas, tais como tristeza, raiva, ansiedade, frustração, insegurança e medo (Costa \& McCrae, 1992). As pessoas com altos escores na dimensão de neuroticismo tendem à hipersensibilidade emotiva. São, geralmente, ansiosas, preocupadas, sensíveis ao ridículo, incapazes de lidar com a pressão, entram facilmente em pânico em situações de emergência. Muitas vezes, quando frustradas, podem reagir de forma agressiva e hostil. De acordo com Eysenck (1959), o neuroticismo é o fator da personalidade que pode ser mensurado com a mesma validade e confiabilidade que a inteligência. Além da recorrência como dimensão universal da personalidade nos diversos sistemas psicométricos e psicobiológicos (Matthews et al., 2003), o neuroticismo parece ter importância fundamental no funcionamento adaptativo das pessoas, em qualquer fase do ciclo vital em que elas se encontrem. Measelle et al. (2005) mostram que avaliações do neuroticismo de crianças de cinco anos de idade, feitas pelos pais ou por meio de autorrelatos, associam-se com medidas laboratoriais de ansiedade após dois anos. De forma semelhante, Shiner e Caspi (2003) afirmam que a tendência a experienciar emoções negativas na infância torna-se um fator de vulnerabilidade para o desenvolvimento de vários tipos de patologias adultas, tais como os transtornos de humor e de ansiedade. Os mesmos autores afirmam, ainda, que altos níveis de ansiedade na infância podem levar as crianças a se afastarem do contato com pares por enxergar a si mesmas como socialmente inadequadas.
Sisto, Bueno e Rueda (2003) verificaram se a aceitação-rejeição de pares está relacionada a traços de personalidade em crianças brasileiras de sete a nove anos de idade. Os principais resultados evidenciam que quanto maior a pontuação em neuroticismo, maior o índice de rejeição entre meninos nas atividades lúdicas. Barbaranelli, Caprara, Rabasca, e Pastorelli (2003) mostraram que o neuroticismo apresenta alta relação com transtornos internalizantes, por exemplo, ansiedade e fobias, e com comportamento agressivo em crianças de 8 a 10 anos. Já no estudo de Romero, Luengo, Gómez-Fraguela e Sobral (2002), extroversão apresenta maior associação com medidas de afeto positivo, enquanto o neuroticismo é o fator mais relacionado ao afeto negativo e a uma pouca satisfação com a vida.

O neuroticismo é, também, constantemente associado a diversas variáveis sociais, como divórcio, instabilidade no casamento, perda salarial, suicídio e consumo de álcool (Nyhus \& Pons, 2005; van Dam, Janssens \& De Bruyn, 2005; White, S. Hendrick \& C. Hendrick, 2004). No que se refere à saúde física, o neuroticismo é frequentemente associado a transtornos nutricionais, especialmente sobrepeso e obesidade (Faith, Flint, Faiburn, Goodwin \& Allison, 2001; Miller, Schmidt, Vaiilancourt, McDougall \& Lalibert, 2006; Nederkoorn, Braet, van Eijs, Tanghe \& Cansen, 2006). A World Health Organization (2005) afirma que crianças obesas são mais propensas a sentirem solidão, tristeza, desesperança e nervosismo, características essas presentes em indivíduos com alto neuroticismo.

No que concerne à educação, diversas investigações encontraram associação negativa entre o neuroticismo e o desempenho escolar (Chamorro-Premuzic \& Furnham, 2003). Chamorro-Premuzic, Furnham e Petrides (2006) argumentam que a associação negativa existente entre o neuroticismo e o desempenho escolar se deve ao fato de que indivíduos altamente neuróticos tendem a experimentar grande ansiedade em situações de testagem, prejudicando seu desempenho em exames e testes escolares. Contudo, Brakto, Chamorro-Premuzic e Saks (2006) acreditam que em universitários os altos níveis de preocupação e de perfeccionismo que caracterizam o indivíduo neurótico podem levá-lo a uma melhor preparação para os exames e, portanto, melhor desempenho. A discrepância entre universitários e crianças pode ser devido ao fato de que os primeiros 
tenham maior habilidade em controlar o seu traço para se adaptar funcionalmente ao ambiente. Chamorro-Premuzic, Furnham, Dissou e Heaven (2005) acreditam, ainda, que o desempenho dos indivíduos em exames escolares pode estar mediado pela relação existente entre as características de personalidade e o tipo de avaliação utilizado em sala de aula. Em específico, eles apontam que indivíduos com elevado neuroticismo se saem pior em exames orais ou em avaliações contínuas.

No Brasil, Sisto et al. (2004) verificaram relação entre traços de personalidade e desenvolvimento visomotor, avaliada pelo teste gestáltico visomotor de Bender, em crianças de seis a doze anos. Um dos resultados principais aponta para a diminuição da integração visomotora com o aumento do neuroticismo.

Deve-se destacar, contudo, que a maioria dos estudos sobre a personalidade na infância que utilizam o modelo dos Cinco Grandes Fatores (CGF) é marcada pela utilização unicamente das descrições que as crianças fazem de si mesmas. Poucos são os estudos que utilizam avaliações multi-informes, provavelmente devido ao alto custo financeiro e temporal que tal procedimento acarreta aos investigadores. No entanto, esta é a clássica orientação do psicometrista e diferencialista Raymond Cattell: dados Q (questionários), T (testes laboratoriais) e L (Life-dados do quotidiano) devem ser integrados para obter maior consistência na interpretação da personalidade (Olea \& Abad, 2006). O presente estudo pretende atender justamente essa orientação por meio de medidas de autorrelato, de heterorrelato feito mediante o relato de pais e de professores -, de entrevista semiestruturada e de observação do comportamento em situação real para a investigação da personalidade em crianças brasileiras.

\section{Método}

\section{Participantes}

A amostra foi composta de 368 sujeitos matriculados da $3^{\text {a a }} 8^{a}$ série e provenientes de uma escola pública federal de ensino fundamental, localizada na cidade de Belo Horizonte (MG). O sexo masculino representou 52,2\% ( $n=192)$ da amostra, idade Média (M) de 11,46 anos e Desvio-Padrão (DP) de 1,78.

\section{Instrumentos}

- Big Five Questionnaire for Children (BFQ-C) versão de autoavaliação (Barbaranelli et al., 1998): visa a mensurar as cinco dimensões da personalidade - Extroversão, Neuroticismo, Cordialidade, Responsabilidade, Abertura a Experiências - em crianças e adolescentes. O instrumento utilizado no presente estudo está em processo de adaptação para o contexto brasileiro pelo Laboratório de Avaliação das Diferenças Individuais (LADI) (www. fafich.ufmg.br/ ladi) e consta de uma versão única para crianças de nove a quinze anos, composta de 75 itens. As respostas são dadas em uma escala do tipo Likert de cinco pontos para todas as faixas etárias.

- Big Five Questionnaire for Children - versão de heteroavaliação adaptada: versão também em processo de validação pelo LADI, constando de 75 itens semeIhantes aos da versão autoavaliação; os itens são constituídos de frases construídas na terceira pessoa (ex. Ele fica nervoso....) e respondidos com base numa escala do tipo Likert de cinco pontos. Para a presente investigação, a versão aplicada a pais e a professores incluiu apenas itens do fator neuroticismo (N), uma vez que os objetivos do estudo estão centrados na avaliação desse fator.

-Eysenck Personality Questionnaire Junior (EPQ-J): medida de autoavaliação da personalidade baseada no Modelo dos Três Fatores de Hans Eysenck (H.J. Eysenck \& S.G. Eysenck, 1998) e que também está em processo de adaptação brasileira pelo LADI. O instrumento, destinado a crianças e adolescentes de 8 a 15 anos de idade, é composto por quatro escalas: Neuroticismo, Extroversão, Psicoticismo e Escala de Sinceridade, perfazendo um total de 81 itens.

- Roteiro de Entrevista Semiestruturada: o objetivo da criação da entrevista semiestruturada foi desenvolver uma abordagem flexível de relato das características de personalidade das crianças, mantendo, contudo, certo grau de estruturação para pontuar as respostas. Nesse sentido, o roteiro de entrevista avalia as principais características comportamentais da dimensão Neuroticismo: Hostilidade, Impulsividade, Ansiedade, Depressão, Constrangimento e Vulnerabilidade. Cada uma dessas características é representada figurativamente para a criança com dois ou mais personagens de desenhos animados ou personalidades que são consideradas representantes extremos de cada um dos 
polos das características. O examinador pontua a resposta dada pela criança com base numa escala do tipo Likert de cinco pontos: de 1 (baixa intensidade do traço) a 5 (elevada intensidade do traço).

- Roteiro de Observação do Comportamento em Grupo: criado com o objetivo de verificar como as crianças manifestam comportamentalmente os traços de personalidade, em especial o de Neuroticismo. Partiu-se do pressuposto de que, na escola, as crianças lidam com demandas e conflitos sociais variados, sendo, portanto, ambiente privilegiado para a observação dos traços de personalidade. O roteiro criado pretendeu avaliar as seguintes características do Neuroticismo: Hostilidade, Impulsividade, Ansiedade, Depressão, Constrangimento e Vulnerabilidade. O examinador pontuava cada uma das características com base numa escala do tipo Likert de cinco pontos: de 1 (baixa intensidade do traço) a 5 (elevada intensidade do traço).

\section{Procedimentos}

A presente investigação constou de duas fases ${ }^{3}$ : na primeira, as crianças responderam, em sala de aula, aos questionários de autorrelato da personalidade; na segunda fase, uma amostra-alvo foi submetida à entrevista semiestruturada e às observações comportamentais no ambiente escolar.

A segunda fase da pesquisa se dividiu em três etapas: 1) construção e aplicação da entrevista semiestruturada; 2) envio e solicitação de preenchimento do questionário de heteroavaliação da personalidade a pais e professores, e 3) observação do comportamento das crianças em contexto escolar.

Para a construção do roteiro da entrevista semiestruturada, o primeiro passo foi fazer uma busca por personagens e personalidades que representassem estímulos familiares para as crianças brasileiras e que tivessem, ao mesmo tempo, as principais características do Neuroticismo de forma bastante explícita para facilitar o reconhecimento. Foram escolhidos dez pares de personagens e personalidades, sendo cada membro do par representante de um dos extremos das características de personalidade. Somente foram escolhidas personagens e personalidades que são, de forma geral, igualmente importantes e centrais em suas atividades cotidianas e em seus papéis nos desenhos animados. Esse cuidado foi tomado na tentativa de minimizar a desejabilidade social. Em seguida, foi realizado um estudo sobre a familiaridade dos estímulos escolhidos com um grupo de 20 crianças, de ambos os sexos, de oito anos de idade, provenientes de uma escola da rede pública estadual da periferia de Belo Horizonte.

A opção por crianças de baixa renda se deu pelo fato de elas terem um acesso mais restrito à internet, ao cinema e à televisão. Dessa forma, pensou-se que se ocorresse uma elevada familiaridade dos estímulos para essas crianças, então seria possível generalizar para outras mais abastadas. Foi solicitado às crianças que indicassem se conheciam ou não aquela figura, de onde a conheciam e suas principais características. A figura era tida como sendo altamente familiar quando $80 \%$ ou mais das crianças a reconheciam e a descreviam corretamente. Sete pares apresentados atingiram o critério estabelecido. No caso de pares formados por seres humanos ou personagens representando seres humanos, optou-se por colocar um representante do sexo masculino e um do sexo feminino para cada extremo da característica (por exemplo: Popeye e Velma X Olívia Palito e Salsicha como representantes da característica Vulnerabilidade), minimizando possíveis efeitos de gênero. Após modificações e ajustes, o instrumento final ficou composto de sete pares, distribuídos ao longo dos seis itens do instrumento.

Após a criação do texto descritivo para cada um dos membros dos pares de figuras de personalidades e personagens de desenhos animados, do exemplo e do texto de rapport, o roteiro da Entrevista Semiestruturada, em sua versão final, foi aplicado para cada criança individualmente, em sessões de uma hora de duração. As entrevistas foram realizadas em horário extraclasse nas dependências do Serviço de Psicologia Aplicada (SPA) da UFMG. Simultaneamente às avaliações das crianças pela entrevista semiestruturada, o Big Five Questionnaire for Children - versão de heterorrelato - foi distribuído aos professores das $3^{\text {as }}, 4^{\text {as }}$ e $5^{\text {as }}$ séries, ou seja, apenas aos professores das séries dos alunos que fizeram a

3183 Procedimentos éticos do projeto aprovados pelo sistema SISNEP, em 2006 [CAE.0459.0.203.000-06]. 
entrevista semiestruturada. De maneira semelhante, foi solicitado aos pais das crianças avaliadas pelos professores que respondessem também à versão adaptada da heteroavaliação do BFQ-C. O instrumento foi enviado aos pais em um envelope lacrado juntamente com uma carta que explica sua finalidade.

Para a observação do comportamento na escola, os professores foram avisados para não alterarem seus comportamentos naturais devido à presença do examinador. Às crianças foi dito que estagiários de psicologia acompanhariam as aulas no período supracitado. Os examinadores foram treinados quanto aos tipos de comportamento a serem observados como característicos da dimensão-alvo, tais como expressar raiva e frustração ao perder para o colega em disputa desportiva ou em sala de aula (hostilidade); interromper a conversa de dois colegas ou a explicação do professor para pedir alguma coisa ou emitir uma opinião, furar a fila da merenda (impulsividade); não mostrar iniciativa em tarefas em grupos ou percepção das brincadeiras ou tarefas em classe (engraçadas, chatas, legais), sendo dependente da avaliação do grupo (vulnerabilidade); mostrar vergonha ao ser repreendido pelo professor ou ao ser solicitado a realizar um exercício na frente de toda classe (constrangimento); entrar para a sala de aula logo após o sinal por medo de que a professora entre na sala antes ou apreensão quando diante de uma avaliação, prova ou outras atividades (ansiedade). Para tanto, utilizaram-se vídeos de filmes ou documentários para realização de avaliações independentes e posterior análise de concordância.

Todos os examinadores já tinham experiência prévia com crianças por meio da realização de estágios curriculares ou extracurriculares em clínicas e escolas e da participação em projetos de pesquisas cujos participantes eram, em sua maioria, crianças. Cada criança foi observada três vezes em dias diferenciados e em aulas diferentes. A observação do comportamento em três momentos distintos foi importante para minimizar os efeitos da presença do avaliador, visto como estímulo novo. Cada examinador observou no máximo cinco crianças por dia. Em virtude da dificuldade em conciliar os horários disponibilizados pela escola para observação do comportamento das crianças com o horário dos examinadores, a mesma criança não pôde ser observada pelo mesmo examinador nos três diferentes dias de observação.

\section{Resultados}

A fim de obter informação sobre as relações existentes entre os itens e, portanto, explorar a estrutura subjacente (latente) às escalas empregadas, realizou-se uma análise fatorial exploratória para cada um dos instrumentos separadamente. Para tanto, utilizou-se uma extração de fatores por meio da técnica Maximum Likelihood (ML) para um fator, isto é, os itens de cada fator teórico tanto do EPQ-J quanto do BFQ-C. Essa técnica foi utilizada para verificar a unidimensionalidade de cada um dos fatores de ambos os instrumentos. Eliminaram-se os itens cujo peso fatorial foi menor do que 20 em seu fator teórico, o qual reduziu para 48 itens no caso do EPQ-J e para 70 itens no caso do BFC.

Em seguida, o conjunto de itens válidos (aqueles que após a análise fatorial mantiveram sua principal carga no fator teórico esperado) de todo o instrumento foi submetido a uma técnica conhecida como Comprehensive Exploratory Factor Analysis com rotação target ou chamada também de rotação procrusteana (Browne, Cudeck, Tateneni \& Mels, 2004). Essa técnica é especial para estruturas fatoriais complexas, em que fatores podem ter cargas secundárias em outros fatores. Essa situação é muito comum em testes de personalidade, cujos fatores dificilmente são totalmente ortogonais e, por tanto, suas correlações dificilmente são iguais a zero. No caso do EPQ-J, os parâmetros de ajuste foram adequados: Chi-square $=3195,49 ; d f=2.012$; Chi/Dif=1.588; RMSEA=0.038., e, no caso do BFC-Q, os parâmetros de ajuste do modelo também se mostraram adequados: Chi-square $=3475,92 ; d f=2075 ;$ Chi/Dif $=1,675$; RMSEA $=0,041$. Os índices de consistência interna para as escalas do EPQ-J foram 0,780 para Neuroticismo (N), 0,647 para Extroversão (E), 0,718 para Psicoticismo (P) e 0,787 para Sinceridade (S). No caso do BFC-Q, os índices alfas foram 0,792 para Abertura a Experiências (A), 0,827 para Cordialidade (C), 0,743 para Extroversão, 0,816 para Neuroticismo e 0,859 para Responsabilidade (R).

Para verificar a existência de validade de construto do Neuroticismo avaliado pelo BFQ-C e pelo EPQ-J, os itens de $\mathrm{N}$ fatorialmente válidos e transformados a $z$ de ambas as escalas foram correlacionados. A correlação entre o $\mathrm{N}$ medido pelos dois instrumentos foi moderada e significativa $(r=0,521 ; p=0,000)$. $O$ valor de " $r$ " encontrado indica que embora haja uma clara 
correspondência entre o $\mathrm{N}$ das duas escalas, não se pode afirmar que elas estejam medindo construtos idênticos.

Com o intuito de selecionar de maneira mais precisa um grupo de crianças com alto e baixo escore em $\mathrm{N}$ e que pudesse ser avaliado mediante outras fontes, a matriz de dados dos dois instrumentos de autoavaliação da personalidade foi submetida a uma nova análise fatorial, utilizando o método de extração ML e rotação Oblimin com Kaiser. Seria possível, assim, extrair os itens de $\mathrm{N}$ fatorialmente válidos para os dois instrumentos. Os índices de ajuste da saída fatorial mostraram-se adequados: Chi-square $=8.825,8 ; d f=6.323$; Chi/ Diff=1.395; RMSEA=0.04. Esse último parâmetro foi calculado com base na fórmula: $\left.R M S E A=\sqrt{ }\left[\chi^{2} / \mathrm{df}-1\right) /(n-1)\right]$, sendo " $n$ " o tamanho da amostra e $d f$ os graus de liberdade (degrees of freedom) do modelo.

No Anexo 1, observa-se a solução de cinco fatores: Abertura Mental (Componente 1), Neuroticismo (Componente 2), Extroversão (Componente 3), Cordialidade/negativo + Psicoticismo/positivo (Componente 4) e Responsabilidade (Componente 5). Dos 30 itens de Neuroticismo dos dois instrumentos, 23 foram fatorialmente válidos. Dessa forma, os 23 itens de $\mathrm{N}$ dos dois instrumentos que se apresentaram válidos fatorialmente passaram a compor o referencial para selecionar as crianças de baixos e altos N. Para tal, somaram-se os itens e transformou-se o resultado em escore-Z e logo a quartil. Obtiveram-se 50 crianças do quartil 1 ( $M=34,39$, $D P=2,99)$ e 42 crianças do quartil $4(M=57,63, D P=4,88)$. Apenas as crianças de $3^{a}$ a $5^{a}$ séries foram selecionadas para compor os quartis 1 e 4.

As 92 crianças com alto e baixo $\mathrm{N}$ foram selecionadas para participarem da entrevista semiestruturada, observação do comportamento em contexto escolar e avaliação da personalidade feita por pais e professores. Contudo, apenas $76,4 \%$ (68) dos pais autorizaram a participação do filho nas atividades citadas acima. Assim sendo, as resultados descritos a seguir estão baseados numa amostra de 68 crianças.

Com o intuito de verificar a associação existente entre o N calculado com base na solução fatorial conjunta do BFQ-C e EPQ-J e o N resultado do somatório dos itens da entrevista semiestruturada, empregou-se a análise de correlação de Spearman, haja vista o tamanho amostral $(n=68)$. Os resultados encontrados apontam para a existência de associação positiva e significativa $(r=0,266 ; p<0,05)$, embora moderada, entre o $N$ avaliado por duas formas distintas, o autorrelato e a entrevista semiestruturada.

O coeficiente de correlação de Spearman também foi utilizado para verificar o grau de associação entre o Neuroticismo (BFQ-C + EPQ-J), avaliado pelo autorrelato, o Neuroticismo avaliado pelos pais e o Neuroticismo avaliado pelos professores. A amostra considerada para essa análise foi de 66 crianças avaliadas por meio de todas as medidas consideradas. Os resultados encontrados apontam que o autorrelato de $\mathrm{N}$ correlacionou-se moderada e positivamente com o relato de $N$ feito pelos pais $(r=0,400 ; p<0,01)$ e também com o relato de $N$ feito pelos professores $(r=0,396$; $p<0,01)$. Contudo, não foi encontrada associação significativa entre o relato de $\mathrm{N}$ feito por pais e por professores.

Para verificar a existência de associação entre as diversas fontes de informação de $\mathrm{N}$ (autorrelato das crianças, relato de pais e relato dos professores) e a observação comportamental, realizou-se uma correlação de Spearman dado o tamanho amostral $(n=66)$. A pontuação em cada uma das seis características de $\mathrm{N}$ consideradas (ver Procedimento) foi somada para obtenção de um escore bruto total na observação. A pontuação bruta para cada um dos três dias da observação foi somada e calculou-se a média para os três dias de observação. Os resultados das correlações realizadas mostraram não haver associação entre a pontuação de N obtida pela observação comportamental e o autorrerelato $(p=0,703)$, o relato dos pais $(p=0,603)$ e o relato dos professores $(p=0,867)$.

Por outro lado, para verificar se as médias da pontuação na observação comportamental foram diferentes para os dois grupos extremos de N (Q1 e Q4), realizou-se a prova $U$ de Mann-Whitney para amostras independentes, haja vista que a variável dependente (pontuação na observação) não possui normalidade. De forma semelhante, o teste de Mann-Whitney não mostrou diferenças significativas das pontuações de $\mathrm{N}$ na observação para os dois quartis $(U=0,524 ; p=0,922)$, indicando que a observação não conseguiu discriminar entre as crianças com alto $\mathrm{N}$ e aquelas com baixo $\mathrm{N}$.

\section{Discussão}

O primeiro passo adotado foi examinar as propriedades psicométricas dos instrumentos de 
autorrelatos da personalidade utilizados no estudo: o BFQ-C e o EPQ-J. O número de fatores encontrados em cada instrumento foi o teoricamente esperado.

De especial interesse para os objetivos do presente trabalho, os parâmetros psicométricos para a dimensão de Neuroticismo nos dois instrumentos de autorrelato foram satisfatórios. Esse resultado indica que o Neuroticismo evidencia-se claramente, confirmando o resultado de outros estudos de que esse fator parece representar uma dimensão estável da personalidade e que possui robustez entre instrumentos e idades.

Devido ao fato de ambos os instrumentos de autorrelato mensurarem o Neuroticismo adequadamente, o passo seguinte foi verificar a validade convergente de N, ou seja, o grau de semelhança de N nas duas escalas de personalidade. A correlação entre o $\mathrm{N}$ dos dois instrumentos foi moderada, apontando para uma considerável similaridade entre as escalas, conforme se observa em estudos com população infantil que utilizam os instrumentos (Barbaranelli et al., 2003; Muris et al., 2005). A similaridade não é maior possivelmente pelo fato de os dois instrumentos operacionalizarem modelos teóricos que divergem levemente quanto à conceitualização de cada um dos fatores (Scholte \& De Bruyn, 2004). Muito embora algumas características de $\mathrm{N}$, tais como labilidade emocional, vulnerabilidade e tendência a experienciar emoções negativas, sejam comuns aos dois modelos, outras não o são. Por exemplo, no modelo CGF, fazem parte da dimensão de Neuroticismo características como a hostilidade (raiva decorrente de frustração) e a impulsividade (incapacidade de inibir respostas comportamentais), que no modelo de Eysenck são definidas de forma diferente, migrando para outras dimensões (Garcia, 2006).

De qualquer modo, aspectos de $\mathrm{N}$ comuns às duas escalas permitiram a seleção de crianças com alto e baixo $\mathrm{N}$ de forma a comparar seus autorrelatos com as demais avaliações. Os resultados encontrados indicam a existência de associação positiva e significativa entre o $\mathrm{N}$, medido pelo autorrelato, e o N, medido pela entrevista. Embora a correlação encontrada entre as medidas tenha sido significativa, a magnitude de tal associação foi pequena. Resultados semelhantes têm sido encontrados em outros estudos que buscam verificar a equivalência de diferentes métodos de avaliação de características da personalidade (P. M. Markey, C. N.
Markey, \&Tinsley, 2004; Reynolds, Ortengren, Richards \& de Wit, 2006).

Embora autorrelato e entrevista não estejam avaliando exatamente os mesmos comportamentos relacionados a N, a entrevista semiestruturada é capaz de capturar a variabilidade existente em $\mathrm{N}$ e discriminar adequadamente crianças com altos e baixos $\mathrm{N}$ nos extremos. Assim sendo, a estratégia de entrevista proposta no presente estudo pode ser considerada um instrumento útil para complementar a avaliação da personalidade em crianças, embora outros estudos com amostras maiores sejam necessários.

No que se refere à heteroavaliação, o relato de adultos acerca das tendências comportamentais das crianças tem sido utilizado extensivamente tanto na literatura clínica quanto na desenvolvimental. Contudo, os resultados revelam a quase impossibilidade de se encontrar alta concordância entre pais e professores na avaliação comportamental de uma criança (De Fruyt \& Vollrath, 2003; Grietens et al. 2004).

No presente estudo, os resultados indicam a existência de associação positiva e significativa, embora moderada, entre o autorrelato e a avaliação dos pais e a dos professores. Resultados similares foram encontrados por Laidra, Allik, M. Harro, Merenäkk e J. Harro (2006) e Barbaranelli et al. (2003). Contudo, a presente investigação não encontrou associação significativa entre o relato dos pais e dos professores para $\mathrm{N}$, indicando que pais e professores possuem dificuldades para concordar com relação aos comportamentos apresentados pela criança. De forma geral, a maior parte dos estudos aponta para a existência de baixos coeficientes de associação, os quais praticamente nunca ultrapassam a barreira de 0,20 entre o relato de pais e professores. Laak, De Goede e Brugman (2001) e Barbaranelli et al. (2003) sugerem que os professores podem não ter acurácia ao estimar traços de personalidade que não sejam diretamente relevantes para o comportamento em sala de aula e para o desempenho escolar ou que sejam difíceis de serem observados no contexto escolar. Já De Fruyt e Vollrath (2003) afirmam que os pais são mais lenientes ao avaliar as características negativas de seus filhos, podendo, com isso, diminuir a acurácia dos relatos dados por eles.

Grande parte dos pesquisadores concorda que as diferenças entre os informantes não devem ser inter- 
pretadas como reflexo da não confiabilidade dos procedimentos de medida ou como havendo um tipo de informante melhor do que o outro. Zucker, M. Morris, Ingram, R.D. Morris e Bakeman (2002) asseveram que a discordância refletiria atitudes, julgamentos e interpretações divergentes dos itens de uma dada escala e do comportamento em si, além dos possíveis vieses do adulto em relação ao sujeito avaliado. Já Mitsis, McKay, Schultz, Newcorn e Halperin (2000) acreditam que a informação sobre o comportamento é única e específica a cada ambiente, seja em casa, seja na escola. Os resultados do presente estudo corroboram essas ideias na medida em que se pode observar associação entre o autorrelato e o relato dado por pais e professores, indicando que diferentes tipos de informantes veem a criança a partir de diferentes perspectivas que não são mutuamente excludentes. A inexistência de associação significativa entre o relato de pais e professores para $\mathrm{N}$, entretanto, indica que novos estudos são necessários com amostras maiores e com maior variabilidade comportamental para verificar a generalidade dos achados.

Finalmente, a observação comportamental de N em contexto escolar não apresentou associação com o autorrelato nem com o relato de pais e professores. Várias podem ter sido as razões pelas quais as observações não se mostraram indicadores satisfatórios das diferenças individuais em Neuroticismo. As observações na escola, por exemplo, foram realizadas em ambientes diversos (sala de aula, recreio, educação física, aulas fora do ambiente convencional) que facilitam a expressão de certas características comportamentais em detrimento de outras. Não obstante, conforme asseveram Reynolds et al. (2006), a observação, quer em situação real, quer em ambiente estruturado, restringe-se a um pequeno número de comportamentos, o que, muitas vezes, diminui ou anula sua associação com os questionários de personalidade.

Com relação à ausência de associação entre as observações e o relato de pais e professores, pode-se especular, ainda, que ela se deva à variação situacional das manifestações do comportamento (Mitsis et al., 2000). No caso dos pais, tem-se que as observações se deram em contexto escolar, sendo, portanto, um ambiente do qual eles têm poucas informações acerca do comportamento de seus filhos. Com respeito aos professores, os aspectos internos de $\mathrm{N}$ são difíceis de serem avaliados na escola, sendo esse um dos motivos pelos quais professores e observadores avaliam o traço com menos acurácia.

A presente investigação buscou responder alguns questionamentos recorrentes, nos últimos anos, na literatura sobre as diferenças individuais em personalidade na infância, principalmente em relação à universalidade e à força da dimensão de Neuroticismo na descrição da personalidade infantil. Buscou-se verificar também a possibilidade de aplicação de outros instrumentos, além dos questionários, para mensuração das diferenças individuais e a concordância entre várias fontes de avaliação.

Uma das limitações, contudo, foi o número amostral pequeno de crianças com altos e baixos $\mathrm{N}$, impossibilitando a utilização de análises estatísticas mais poderosas. Futuras pesquisas que utilizem entrevistas e observações na avaliação da personalidade devem incluir a avaliação da mesma criança por diferentes examinadores com o intuito de verificar o grau de concordância entre eles. As observações e as entrevistas ainda deverão ser filmadas com o intuito de corrigir possíveis erros na avaliação.

Apesar das limitações, a presente investigação fornece evidências de que é possível delinear estudos que incluam fontes diversas de mensuração da personalidade e que essas são, por sua vez, complementares. Ademais, esse estudo traz contribuições importantes para o estudo das diferenças individuais em personalidade infantil, no Brasil, uma vez que apresenta dados até então restritos a populações adultas.

\section{Referências}

Barbaranelli, C., Caprara, G., \& Rabasca, A. (1998). BFQ-C Big Five Questionnaire for Children. Firenze: Organizzazioni Speciali.

Barbaranelli, C., Caprara, G., Rabasca, A., \& Pastorelli, C. (2003). A questionnaire for measuring the Big Five in late childhood. Personality and Individual Differences, 34 (4), 645-664

Bratko, D., Camorro-Prezumic, T., \& Saks, Z. (2006). Personality and school performance: Incremental validity of self - and peer-ratings over intelligence. Personality and Individual Differences, 41 (1), 131-142. 
Browne, M. W., Cudeck, R., Tateneni, K., \& Mels G. (2004). CEFA: Comprehensive Exploratory Factor Analysis, Version 2.00 [Computer software and manual]. Retrieved January 30, 2007, available from http://quantrm2.psy. ohio-state.edu/

Caspi, A., Roberts, B.W. \& Shiner, R.L. (2005). Personality Development: Stability and Change. Annual Review of Psychology, 56, 453-484.

Chamorro-Premuzic, T. \& Furnham, A. (2003). Personality predicts academic performance: evidence from two longitudinal studies on British University students. Journal of Research in Personality, 37 (4), 319-338.

Chamorro-Premuzic, T., Furnham, A., Dissou, G., \& Heaven, P. (2005). Personality and preference for academic assessment: a study with Australian University students. Learning and Individual Differences, 15 (4), 247-256.

Chamorro-Premuzic, T., Furnham, A., \& Petrides, K. (2006). Personality and Intelligence: The relationship of Eysenck's Giant Three with Verbal and Numerical Ability. Journal of Individual Differences, 27 (3), 147-150.

Costa, P. T. Jr., \& McCrae, R.R. (1992). NEO-PI-R: Professional Manual. Odessa, FL: Psychological Assessment Resources.

De Fruyt, F., \& Vollrath, M. (2003). Inter-parent agreement on higher and lower level traits in two countries: effects of parent and child gender. Personality and Individual Differences, 35 (2), 289-301.

Eysenck, H. J. (1959). Estudio cientifico de la personalid. Buenos Aires: Editorial Paidos.

Eysenck, H.J., \& Eysenck, S.G. (1998). Manual of the Eysenck Personality Questionnaire (Junior \& Adult). Adaptado por Nicolas Seisdedos e Agustín Cordero. Madrid: TEA Ediciones.

Faith, M.S., Flint, J., Fairburn, C.G., Goodwin, G.M., \& Allison, D.B. (2001). Gender differences in the relationship between personality dimensions and relative body weight. Obesity Research, 9 (10), 647- 650.

Garcia, L.F. (2006). Teorias psicométricos da personalidade. In: C. Flores-Mendoza \& R. Colom (Orgs.), Introdução à psicologia das diferenças individuais (pp.219-242). Porto Alegre: Artmed.

Grietens, H., Onghena, P., Prinzie, P., Gadeyne, E., Van Assche, V., Ghesquière, P. et al. (2004). Comparison of mothers', fathers', and teachers' reports on problem behavior in 5- to 6-year old children. Journal of Psychopathology and Behavioral Assessment, 2 (2), 137-146.

Halverson, C.F., Havill, V.L., Deal, J., Baker, S.R., Victor, J.B., Pavlopoulos, V., et al. (2003). Personality structure as derived from parental ratings of free descriptions of children: the inventory of child individual differences. Journal of Personality, 71 (6), 995-1026.

Laak, J. J. F., De Goede, M, P. M., \& Brugman, G. M. (2001). Teacher's judgment of pupils: agreement and accuracy. Social Behavior and Personality, 29 (3), 257-270.

Laidra, K., Allik, J., Harro, M., Merenäkk, L. \& Harro, J. (2006). Agreement among adolescents, parents, and teachers on adolescent personality. Assessment, 13 (2), 187-196.
Markey, P. M., Markey, C. N., \& Tinsley, B. J. (2004). Childrens' behavioral manifestations of the five-factor model of personality. personality and Social Psychology Bulletin, 30 (4), 423-432.

Matthews, G., Deary, I.J. \& Whiteman, M.C. (2003). Personality traits ( $2^{\text {nd }}$ ed.) London: Cambridge University Press.

Measelle, J.R., John, O.P., Ablow, J.C., Cowan, P.A., \& Cowan, C.P. (2005). Can children provide coherent, stable, and valid self-reports on the big five dimensions? A longitudinal study from ages 5 to 7 . Journal of Personality and Social Psychology, 89 (1), 90-106.

Mervielde, I., Buyst, V., \& De Fruyt, F. (1995). The Validity of the big-five as a model for Teachers' ratings of Individual differences among children aged 4-12 years. Personality and Individual Differences, 18 (4), 525-534.

Miller, J. L., Schmidt, L. A., Vaiilancourt, T., McDougall, P., \& Lalibert, M. (2006). Neuroticism and Introversion: a risky combination for disordered among a non-clinical sample of undergraduate women. Eating Behaviors, 7 (1), 69-78.

Mitsis, E.M., McKay, K.E., Schultz, K.P., Newcorn, J.H., \& Halperin, J.M. (2000). Parent-teacher concordance for DSM-IV attention-deficit/hyperactivity disorder in a clinic-referred sample. Journal of the American Academy of Child and Adolescent Psychology, 39 (3), 308-313.

Muris, P., Meesters, C., \& Diederen, R. (2005). Psychometric Properties of the Big Five Questionnaire for Children (BFQ-C) in a Dutch sample of young adolescents. Personality and Individual Differences, 38 (8), 1757- 1769.

Nederkoorn, C., Braet, C., Van Eijs, W., Tanghe, A., \& Cansen, A. (2006). Why obese children cannot resist the food? The role of impulsivity. Eating Behaviors, 7 (4),315-322.

Nyhus, E. K., \& Pons, E. (2005). The effect of personality on earnings. Journal of Economic Psychology, 26 (3), 363-384.

Olea, J. \& Abad, F.J. (2006). A medição das diferenças individuais. In C. Flores-Mendoza \& R. Colom (Orgs.), Introdução à psicologia das diferenças individuais (pp.325-354). Porto Alegre: Artmed.

Reynolds, B., Ortengren, A., Richards, J.B., \& de Wit, H. (2006). Dimensions of impulsive behavior: personality and behavioral measures. Personality and Individual Differences, 40 (2), 305-315.

Romero, E., Luengo, M.A., Gómez-Fraguela, J.A., \& Sobral, J. (2002). La estructura de los rasgos de personalidad en adolescents: el modelo de cinco factores y los cinco alternativos. Psicothema, 14 (1), 134-143.

Scholte, R.H.J., \& De Bruyn, E.E.J. (2004). Comparison of the giant three and the big five in early adolescents. Personality and Individual Differences, 36 (6), 1353-1357.

Shiner, R. L. (1998). How shall we speak of children's personalities in middle childhood? A preliminary taxonomy. Psychological Bulletin, 124 (3), 308-332.

Shiner, R. L., \& Caspi, A. (2003). Personality differences in childhood and adolescence: measurement, development, 
and consequences. Journal of Child Psychology and Psychiatry, 44 (1), 2-32.

Sisto, F. F., Bueno, J. M. H. \& Rueda, F. J. M. (2003). Traços de personalidade na infância e distorção e integração de formas: um estudo de validade. Psicologia em Estudos, 8 $(1), 77-84$

Sisto, F. F., Oliveira, S. M. S.S., Oliveira, K. L., Bartholomeu, D., Oliveira, J. C. S., \& Costa, O. R. S. (2004). Escala de traços de personalidade para crianças e aceitação social entre pares. Interação em Psicologia, 8 (1), 15-24.

van Dam, C., Janssens, J.M.A.M., \& De Bruyn, E.E.J. (2005). PEN, Big Five, juvenile delinquency and criminal recidivism. Personality and Individual Differences, 39 (1), 7-15.
White, J. K., Hendrick, S.S, \& Hendrick, C. (2004). Big five personality variables and relationship constructs. Personality and Individual Differences, 37 (7), 1519-1530.

World Health Organization. (2005).Childhood Obesity. Retrieved January 30, 2007, available from http:// www.paho.org/english/cfni/home.htm

Zucker, M., Morris, M. Ingram, S.M., Morris, R.D., \& Bakeman, R. (2002). Concordance of self- and informant ratings of adults' current and childhood attention-deficit/ hyperactivity disorder symptoms. Psychological Assessment, 14 (4), 379-389.

Received on: 3/4/2008

Final version resubmitted on: 24/4/2009

Approved on: 26/5/2009 
ANEXO 1

SOLUÇÃO FATORIAL CONJUNTA DO BFQ-C + EPQ-J

\begin{tabular}{|c|c|c|c|c|c|}
\hline Itens & A & $\mathrm{N}$ & $E$ & $-C /+P$ & $\mathrm{R}$ \\
\hline bfc_5_a1 & 0,42 & 0,03 & 0,07 & 0,01 & 0,09 \\
\hline bfc_10_a2 & 0,37 & $-0,04$ & 0,15 & 0,02 & 0,17 \\
\hline bfc_12_a3 & 0,61 & $-0,13$ & $-0,16$ & 0,14 & 0,15 \\
\hline bfc_18_a4 & 0,64 & $-0,05$ & $-0,09$ & $-0,01$ & 0,00 \\
\hline bfc_24_a5 & 0,10 & 0,03 & 0,09 & $-0,15$ & 0,42 \\
\hline bfc_30_a6 & 0,58 & $-0,10$ & $-0,12$ & 0,09 & 0,16 \\
\hline bfc_33_a7 & 0,15 & $-0,03$ & 0,15 & $-0,07$ & 0,19 \\
\hline bfc_36_a8 & 0,13 & $-0,05$ & 0,15 & 0,07 & 0,27 \\
\hline bfc_43_a9 & 0,28 & $-0,03$ & 0,18 & 0,16 & 0,17 \\
\hline bfc_46_a10 & 0,27 & $-0,09$ & $-0,04$ & 0,12 & 0,21 \\
\hline bfc_52_a11 & 0,18 & $-0,02$ & 0,25 & $-0,12$ & 0,22 \\
\hline bfc_59_a12 & 0,04 & $-0,05$ & 0,26 & $-0,06$ & 0,25 \\
\hline bfc_62_a13 & 0,65 & $-0,11$ & $-0,07$ & 0,06 & 0,09 \\
\hline bfc_67_a14 & 0,18 & 0,06 & 0,41 & $-0,07$ & 0,10 \\
\hline bfc_2_cl & 0,21 & 0,03 & 0,20 & $-0,12$ & 0,04 \\
\hline bfc_11_c2 & 0,38 & 0,03 & $-0,02$ & $-0,37$ & 0,04 \\
\hline bfc_13_c3 & 0,43 & 0,08 & 0,12 & $-0,10$ & $-0,07$ \\
\hline bfc_16_c4 & 0,10 & 0,19 & 0,20 & $-0,18$ & 0,21 \\
\hline bfc_21_c5 & 0,19 & 0,02 & 0,18 & $-0,28$ & $-0,03$ \\
\hline bfc_27_c6 & 0,19 & 0,01 & 0,17 & $-0,43$ & 0,02 \\
\hline bfc_32_c7 & 0,20 & $-0,01$ & 0,11 & $-0,42$ & 0,02 \\
\hline bfc_38_c8 & 0,15 & $-0,19$ & $-0,04$ & $-0,26$ & 0,02 \\
\hline bfc_45_c9 & 0,32 & 0,01 & 0,22 & $-0,14$ & 0,17 \\
\hline bfc_47_c10 & 0,14 & 0,03 & 0,37 & $-0,07$ & 0,01 \\
\hline bfc_51_c11 & 0,20 & 0,09 & 0,05 & $-0,27$ & 0,19 \\
\hline bfc_60_c12 & 0,04 & 0,04 & 0,25 & $-0,05$ & 0,26 \\
\hline bfc_64_c13 & 0,37 & $-0,06$ & 0,28 & $-0,11$ & $-0,17$ \\
\hline bfc_69_c14 & 0,16 & $-0,01$ & 0,40 & 0,05 & 0,28 \\
\hline bfc_70_c15 & 0,23 & 0,10 & 0,27 & $-0,20$ & 0,07 \\
\hline bfc_1_e1 & 0,20 & 0,16 & 0,29 & $-0,12$ & $-0,17$ \\
\hline bfc_14_e3 & 0,10 & $-0,05$ & 0,42 & 0,10 & 0,11 \\
\hline bfc_19_e4 & 0,21 & 0,13 & 0,46 & $-0,14$ & $-0,07$ \\
\hline bfc_23_e5 & 0,46 & 0,09 & 0,06 & 0,03 & $-0,02$ \\
\hline bfc_40_e7 & 0,20 & 0,03 & 0,46 & 0,01 & $-0,13$ \\
\hline bfc_42_e8 & 0,45 & $-0,02$ & 0,07 & 0,09 & $-0,06$ \\
\hline bfc_50_e9 & 0,47 & $-0,02$ & 0,09 & $-0,09$ & 0,00 \\
\hline bfc_55_e10 & $-0,02$ & $-0,05$ & 0,46 & 0,11 & 0,02 \\
\hline bfc_57_e11 & 0,27 & $-0,07$ & 0,41 & $-0,03$ & $-0,05$ \\
\hline bfc_63_e12 & 0,20 & $-0,01$ & 0,41 & 0,16 & 0,20 \\
\hline bfc_71_e13 & 0,03 & 0,09 & 0,45 & 0,03 & 0,01 \\
\hline bfc_72_e14 & $-0,01$ & $-0,01$ & 0,27 & 0,09 & 0,05 \\
\hline bfc_73_e15 & 0,29 & 0,07 & 0,25 & $-0,04$ & 0,08 \\
\hline epq_1_e & $-0,05$ & 0,01 & 0,37 & $-0,12$ & $-0,02$ \\
\hline epq9_e & 0,04 & $-0,05$ & 0,29 & $-0,02$ & 0,00 \\
\hline epq_13_e & $-0,06$ & $-0,05$ & 0,29 & $-0,09$ & 0,13 \\
\hline epq_17_e & 0,13 & $-0,15$ & 0,28 & 0,02 & $-0,06$ \\
\hline epq_28_e & $-0,01$ & 0,05 & 0,24 & $-0,10$ & 0,20 \\
\hline epq_33_e & 0,02 & $-0,01$ & 0,22 & $-0,12$ & 0,02 \\
\hline epq_37_e & 0,04 & $-0,08$ & 0,20 & 0,04 & 0,16 \\
\hline epq_41_e & $-0,05$ & $-0,08$ & 0,30 & $-0,04$ & 0,19 \\
\hline epq_44_e & 0,02 & $-0,05$ & 0,30 & $-0,09$ & 0,03 \\
\hline epq48_e & $-0,03$ & $-0,13$ & 0,26 & $-0,16$ & $-0,13$ \\
\hline
\end{tabular}


ANEXO 1

SOLUÇÃO FATORIAL CONJUNTA DO BFQ-C + EPQ-J

Continuação

\begin{tabular}{|c|c|c|c|c|c|}
\hline Itens & A & N & E & $-C /+P$ & R \\
\hline epq_56_e & $-0,18$ & $-0,12$ & 0,16 & $-0,14$ & $-0,04$ \\
\hline epq_61_e & $-0,03$ & $-0,24$ & 0,20 & 0,11 & 0,09 \\
\hline epq_67_e & $-0,03$ & 0,01 & 0,18 & 0,04 & 0,00 \\
\hline epq_70_e & 0,11 & 0,04 & 0,33 & $-0,01$ & $-0,01$ \\
\hline epq_74_e & $-0,16$ & $-0,07$ & 0,34 & $-0,01$ & 0,13 \\
\hline epq76_e & $-0,02$ & $-0,13$ & 0,26 & $-0,17$ & $-0,24$ \\
\hline epq_79_e & 0,01 & $-0,17$ & 0,19 & 0,04 & 0,14 \\
\hline bfc_4_n1 & 0,09 & 0,53 & 0,01 & 0,17 & $-0,12$ \\
\hline bfc_6_n2 & 0,05 & 0,44 & 0,01 & 0,10 & $-0,20$ \\
\hline bfc_8_n3 & $-0,05$ & 0,39 & 0,10 & 0,22 & $-0,05$ \\
\hline bfc_15_n4 & 0,04 & 0,59 & $-0,03$ & 0,18 & $-0,13$ \\
\hline bfc_17_n5 & $-0,04$ & 0,36 & 0,10 & 0,31 & $-0,07$ \\
\hline bfc_29_n7 & $-0,06$ & 0,44 & 0,04 & 0,06 & 0,17 \\
\hline bfc_31_n8 & $-0,04$ & 0,54 & $-0,14$ & $-0,09$ & 0,08 \\
\hline bfc_39_n9 & 0,06 & 0,22 & 0,18 & 0,24 & 0,06 \\
\hline bfc_41_n10 & 0,05 & 0,47 & 0,11 & 0,21 & $-0,01$ \\
\hline bfc_49_n11 & 0,04 & 0,62 & 0,01 & 0,20 & 0,02 \\
\hline bfc_54_n12 & $-0,02$ & 0,20 & 0,33 & 0,33 & $-0,18$ \\
\hline bfc_58_n13 & 0,05 & 0,60 & 0,03 & $-0,23$ & $-0,15$ \\
\hline bfc_61_n14 & 0,17 & 0,38 & 0,13 & $-0,10$ & 0,12 \\
\hline epq_2_n & 0,05 & 0,43 & $-0,10$ & 0,13 & 0,00 \\
\hline epq_6_n & $-0,15$ & 0,24 & $-0,09$ & 0,07 & $-0,07$ \\
\hline epq_10_n & $-0,04$ & 0,32 & 0,03 & 0,05 & 0,07 \\
\hline epq_14_n & $-0,07$ & 0,39 & $-0,02$ & 0,04 & $-0,02$ \\
\hline epq_18_n & 0,02 & 0,55 & 0,08 & $-0,19$ & $-0,13$ \\
\hline epq_22_n & $-0,07$ & 0,41 & $-0,18$ & 0,02 & 0,05 \\
\hline epq_26_n & $-0,07$ & 0,23 & 0,12 & 0,10 & $-0,18$ \\
\hline epq_29_n & $-0,16$ & 0,38 & $-0,04$ & $-0,03$ & 0,19 \\
\hline epq_34_n & $-0,04$ & 0,43 & $-0,17$ & 0,10 & 0,00 \\
\hline epq_45_n & $-0,01$ & 0,57 & $-0,18$ & $-0,17$ & 0,03 \\
\hline epq_49_n & 0,08 & 0,40 & $-0,19$ & 0,11 & $-0,07$ \\
\hline epq_53_n & $-0,03$ & 0,24 & 0,23 & 0,11 & $-0,20$ \\
\hline epq_66_n & $-0,12$ & 0,24 & 0,08 & 0,18 & $-0,35$ \\
\hline epq_68_n & $-0,07$ & 0,35 & 0,04 & $-0,16$ & 0,08 \\
\hline epq_71_n & $-0,11$ & 0,33 & $-0,20$ & 0,03 & 0,10 \\
\hline epq_77_n & 0,06 & 0,56 & 0,01 & $-0,15$ & $-0,15$ \\
\hline epq_80_n & $-0,14$ & 0,25 & 0,10 & 0,02 & 0,17 \\
\hline bfc_3_r1 & 0,22 & $-0,06$ & $-0,11$ & $-0,09$ & 0,34 \\
\hline bfc_7_r2 & 0,19 & 0,10 & 0,03 & $-0,09$ & 0,48 \\
\hline bfc_20_r3 & 0,20 & 0,02 & 0,07 & $-0,04$ & 0,37 \\
\hline bfc_22_r4 & 0,19 & $-0,07$ & $-0,25$ & $-0,15$ & 0,47 \\
\hline bfc_25_r5 & 0,04 & 0,05 & 0,06 & $-0,11$ & 0,54 \\
\hline bfc_28_r6 & 0,24 & $-0,05$ & $-0,08$ & $-0,17$ & 0,34 \\
\hline bfc_34_r7 & 0,46 & $-0,07$ & 0,07 & $-0,05$ & 0,13 \\
\hline bfc_37_r8 & 0,06 & $-0,11$ & 0,05 & $-0,02$ & 0,37 \\
\hline bfc_44_r9 & 0,22 & 0,07 & 0,08 & $-0,02$ & 0,33 \\
\hline bfc_48_r10 & 0,10 & 0,00 & 0,23 & $-0,10$ & 0,40 \\
\hline bfc_53_r11 & $-0,04$ & $-0,04$ & 0,03 & 0,02 & 0,59 \\
\hline bfc_56_r12 & 0,20 & 0,00 & $-0,01$ & $-0,14$ & 0,32 \\
\hline bfc_65_r13 & 0,20 & $-0,09$ & 0,05 & $-0,08$ & 0,36 \\
\hline bfc_74_r14 & 0,11 & 0,05 & 0,05 & 0,02 & 0,43 \\
\hline bfc_75_r15 & 0,09 & 0,02 & 0,03 & $-0,01$ & 0,56 \\
\hline
\end{tabular}


ANEXO 1

SOLUÇÃO FATORIAL CONJUNTA DO BFQ-C + EPQ-J

Conclusão

\begin{tabular}{|c|c|c|c|c|c|}
\hline Itens & A & $N$ & $E$ & $-C /+P$ & $R$ \\
\hline epq_7_p & 0,05 & $-0,06$ & 0,08 & 0,39 & $-0,20$ \\
\hline epq_12_p & $-0,05$ & $-0,03$ & $-0,11$ & 0,47 & 0,02 \\
\hline epq15_p & 0,13 & $-0,03$ & $-0,13$ & 0,21 & 0,05 \\
\hline epq_19_p & $-0,04$ & 0,08 & 0,00 & 0,30 & $-0,18$ \\
\hline epq_23_p & 0,00 & 0,09 & 0,06 & 0,45 & 0,00 \\
\hline epq30_p & 0,06 & $-0,16$ & $-0,15$ & 0,22 & $-0,31$ \\
\hline epq_32_p & 0,06 & 0,28 & 0,06 & 0,13 & $-0,01$ \\
\hline epq_35_p & 0,04 & $-0,02$ & 0,00 & 0,46 & $-0,14$ \\
\hline epq_39_p & 0,05 & 0,24 & 0,05 & 0,38 & $-0,02$ \\
\hline epq_42_p & $-0,03$ & 0,25 & 0,13 & 0,37 & $-0,03$ \\
\hline epq_46_p & $-0,04$ & $-0,08$ & 0,11 & 0,54 & $-0,21$ \\
\hline epq_50_p & $-0,03$ & 0,05 & $-0,04$ & 0,26 & 0,05 \\
\hline epq63_p & 0,10 & $-0,05$ & $-0,23$ & 0,17 & $-0,24$ \\
\hline epq72_p & 0,08 & 0,05 & $-0,10$ & 0,27 & $-0,09$ \\
\hline
\end{tabular}

* A: abertura a experiências; N: neuroticismo; E: extroversão; C: cordialidade; P: Psicoticismo; R: responsabilidade.

** Os itens em negrito são aqueles fatorialmente válidos e que representam o fator teórico (carga >0,25). 